\title{
Anna Candida Felici, Agnese Fusaro, Asma Ibrahim, Khaleemullah Lashari, Niccolò Manassero, Mario Piacentini, Valeria Piacentini Fiorani, Alessandro Tilia. "Archaeological Excavations at Babhore, Sidh. Preliminary Report on the Pakistani-Italian 2014 and 2015 Field Seasons"
}

Julien Cuny

\section{(2) OpenEdition Journals}

\section{Édition électronique}

URL : http://journals.openedition.org/abstractairanica/47217

DOI : 10.4000/abstractairanica. 47217

ISBN : 1961-960X

ISSN : 1961-960X

Éditeur :

CNRS (UMR 7528 Mondes iraniens et indiens), Éditions de l'IFRI

Référence électronique

Julien Cuny, «Anna Candida Felici, Agnese Fusaro, Asma Ibrahim, Khaleemullah Lashari, Niccolò Manassero, Mario Piacentini, Valeria Piacentini Fiorani, Alessandro Tilia. "Archaeological Excavations at Babhore, Sidh. Preliminary Report on the Pakistani-Italian 2014 and 2015 Field Seasons" », Abstracta Iranica [En ligne], Volume 37-38-39 | 2018, document 77, mis en ligne le 30 décembre 2018, consulté le 02 octobre 2020. URL : http://journals.openedition.org/abstractairanica/47217; DOI : https://doi.org/10.4000/abstractairanica.47217

Ce document a été généré automatiquement le 2 octobre 2020.

Tous droits réservés 


\section{Anna Candida Felici, Agnese Fusaro, Asma Ibrahim, Khaleemullah Lashari, Niccolò Manassero, Mario Piacentini, Valeria Piacentini Fiorani, Alessandro Tilia. "Archaeological Excavations at Babhore, Sidh. Preliminary Report on the Pakistani-Italian 2014 and 2015 Field Seasons"}

Julien Cuny

\section{RÉFÉRENCE}

Anna Candida Felici, Agnese Fusaro, Asma Ibrahim, Khaleemullah Lashari, Niccolò Manassero, Mario Piacentini, Valeria Piacentini Fiorani, Alessandro Tilia, "Archaeological Excavations at Babhore, Sidh. Preliminary Report on the PakistaniItalian 2014 and 2015 Field Seasons", Parthica 18, 2016, p. 126-173

Le site archéologique de Banbhore aujourd'hui perdu dans le delta de l'Indus loin de la mer a été une cité et un port florissants, peut-être l'ancienne Deybul ou Debal des sources médiévales mais, selon les fouilles pakistanaises de F.A. Khan dans les années 1960, comme les fouilles récentes (2014-2015) conjointes pakistanaises, italiennes et française. Sont décrits ici seulement les travaux pakistanais-italiens. Le site est occupé 
depuis l'époque sassanide jusqu'au XIIIe s. Cette région à l'embouchure de l'Indus devrait un jour révéler le port de Barbaricum d'Alexandre le Grand que mentionne le Périple de la Mer Erythrée. Les découvertes des fouilles anciennes et les données récentes, encore modestes, montrent sans surprise le rôle qu'a pu jouer Banbhore entre le monde du Moyen-Orient sassanide puis islamique, via le golfe Persique, et l'Asie du sud-est et jusqu'à la Chine. La ville était protégée par une puissante muraille en pierre renforcée de tours, bien conservée; elle semble divisée en deux parties par un long mur, créant ainsi une ville haute, où a été fouillé un " palais » et une ville basse où se succèdent plusieurs niveaux de constructions -, dont plusieurs ateliers.

2 Le mobilier témoigne de la durée de l'occupation et de la diversité des cultures représentées Pour la période sassanide et début de l'époque islamique au VIII ${ }^{\mathrm{e}} \mathrm{s}$. (p. 156-160), des niveaux épais de plusieurs mètres contenaient de l'architecture, ainsi que des céramiques caractéristiques, Indian Polished Ware et une imitation locale de celle-ci, des torpedo jars, ainsi que de l'eggshell ware plutôt à dater du début de l'Islam et une céramique peinte présente sur une très longue période. Les niveaux les plus profonds seraient des IIe et IIIe s. de n.è. Les dimensions du site et la puissance de la stratigraphie nécessiterait la mise en place d'un très important programme dans la durée.

À noter qu'autre article paru la même année (Niccolò Manassero \& Valeria Piacentini Fiorani, "Scavi della Missione dell'Università Cattolica di Milano alla foce dell'Indo: archeologia e storia". Atti della Accademia delle Scienze di Torino. Classe di Scienze Morali, Storiche e Filologiche, 149, 2015, [2016], p. 155-179) double l'article recensé, car il traite du même sujet ; il est cependant plus bref et beaucoup moins illustré.

\section{AUTEURS}

\section{JULIEN CUNY}

Conservateur au Musée du Louvre, Paris 